\title{
COMMENT
}

Check for updates

\section{Haemoperfusion should only be used for COVID-19 in the context of randomized trials}

\author{
Edward G. Clark $\mathbb{1}^{1 凶}$, Swapnil Hiremath ${ }^{1}$, Lauralyn McIntyre ${ }^{2}$, Ron Wald ${ }^{3}$, \\ Gregory L. Hundemer ${ }^{1}$ and Michael Joannidis $\mathbb{D}^{4}$
}

Interest in the use of haemoperfusion for severe COVID-19 has been spurred by anecdotal reports of its efficacy and expert reviews suggesting theoretical benefits. However, on the basis of the limited current evidence, haemoperfusion remains an experimental therapy that should only be applied within the context of well-designed randomized trials.

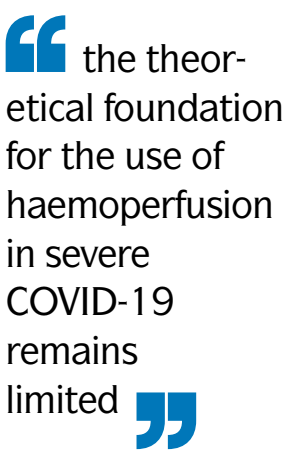

'Division of Nephrology Department of Medicine, The Ottawa Hospital and University of Ottawa, Ottawa, ON, Canada.

${ }^{2}$ Division of Critical Care, Department of Medicine, The Ottawa Hospital and University of Ottawa Ottawa, ON, Canada

${ }^{3}$ Division of Nephrology, St Michael's Hospital and Department of Medicine, University of Toronto, Toronto, ON, Canada

${ }^{4}$ Division of Intensive Care and Emergency Medicine, Department of Internal Medicine, Medical University Innsbruck, Innsbruck, Austria.

凶e-mail:edclark@toh.ca

https://doi.org/10.1038 s41581-020-00352-9
Severe COVID-19 typically presents as acute pneumonia, but some patients develop a dysregulated immune response characteristic of sepsis ${ }^{1}$. In the context of infection, an overwhelming hyperinflammatory response is often termed a 'cytokine storm' or cytokine release syndrome (CRS). CRS is characterized by high levels of circulating pro-inflammatory cytokines (e.g. IL-1 $\beta$, IL-6 and tumour necrosis factor) and is associated with worsening of acute respiratory distress syndrome (ARDS). Although release of pro-inflammatory cytokines can be an appropriate response to severe infection, an exaggerated response is likely responsible for multiple organ dysfunction. High levels of pro-inflammatory cytokines correlate directly with the severity of lung injury in patients with severe COVID-19 (REF. ${ }^{1}$ ), and CRS is associated with multiorgan dysfunction, including acute kidney injury (AKI). CRS results in organ dysfunction and tissue damage by promoting endothelial dysfunction and microvascular and macrovascular thrombosis, which is a widely reported phenomenon in patients with COVID-19 (REF. ${ }^{1}$ ).

Mitigation of inflammation in COVID-19 was recently evaluated in the RECOVERY trial, which assessed the efficacy of dexamethasone in 6,425 patients ${ }^{2}$. Compared with usual care, dexamethasone significantly reduced mortality among patients who were receiving invasive mechanical ventilation $(29.3 \%$ versus $41.4 \%$; rate ratio (RR) 0.64 ; $95 \%$ confidence interval (CI) 0.51 0.81 ). Among those receiving oxygen without invasive mechanical ventilation, dexamethasone was associated with a smaller but significant reduction in mortality (23.3\% versus $26.2 \%$; RR 0.82; 95\% CI $0.72-0.94$ ). By contrast, no mortality benefit and a trend towards worse outcomes with dexamethasone was observed among patients who did not require oxygen. The beneficial effect of dexamethasone in patients with more severe COVID-19 was likely due to immunomodulation of the pro-inflammatory response, particularly in the lungs.

Another potential approach to mitigate excessive inflammation in patients with COVID-19 is the use of haemoperfusion to remove pro-inflammatory cytokines from the blood ${ }^{3,4}$. In this form of extracorporeal organ support, the blood is circulated through a sorbentcontaining cartridge (or column), which non-selectively adsorbs, and therefore removes, larger endogenous and exogenous molecules, usually targeting cytokines, endotoxin, pathogens or a combination of these factors, depending on the type of sorbent (for example, pure resins, polymyxin-coated resins or heparin-coated resins). Interest in the use of haemoperfusion with cartridges that remove cytokines or both cytokines and virus particles for the treatment of severe COVID-19 has been spurred by anecdotal reports of its efficacy and expert reviews highlighting its theoretical benefits $^{3,4}$. The FDA has granted temporary authorization under the Emergency Use Authorization (EUA) programme for four haemoperfusion devices for treatment of patients with severe COVID-19 to mitigate CRS. These devices are designed to specifically eliminate endotoxins, cytokines, pathogens or a combination of these factors ${ }^{5}$. Depending on the device, these haemoperfusion cartridges can be used in conjunction with, or independently of, kidney replacement therapy (KRT), apheresis or extracorporeal membrane oxygenation (ECMO).

Theoretically, haemoperfusion could abrogate or mitigate CRS and the resulting positive feedback loop of immune-mediated inflammation that can lead to increasingly aggressive inflammation and death. Nonetheless, as highlighted in a recent expert review, "the mechanisms by which [haemoperfusion] might exert beneficial effects remain poorly understood" (REF. $\left.{ }^{4}\right)$. Moreover, multiple theoretical considerations 


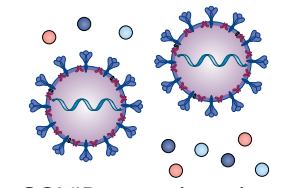

COVID-19 and cytokine release syndrome

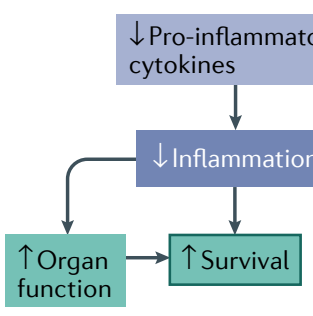

Potential benefits

Potential risks

Fig. 1 Potential risks and benefits of haemoperfusion for cytokine removal in severe COVID-19. Although haemoperfusion can remove cytokines from the blood, evidence suggests that this intervention might not substantially reduce circulating cytokine levels ${ }^{6}$. Moreover, in addition to potential benefits, a non-selective reduction in pro-inflammatory and anti-inflammatory cytokines carries potential risks. Haemoperfusion can also potentially lead to complications that are not directly related to cytokine removal, including hypotension and arrhythmias.

use of uncontrolled interventions during the COVID-19 pandemic ... represents a missed learning opportunity make the broad application of haemoperfusion unlikely to be beneficial in patients with CRS with an infectious cause, including those with COVID-19. Haemoperfusion devices remove both pro-inflammatory and antiinflammatory cytokines. Baseline levels of different cytokines are likely to vary widely between patients and also with the phase and severity of illness. Haemoperfusion could potentially lead to harm either by hindering resolution of excess inflammation (for example, by removing anti-inflammatory mediators as they start to predominate) or conversely by causing excessive immunosuppression (for example, by attenuating an appropriate pro-inflammatory response) such that direct virus-mediated damage is not sufficiently checked. Second, whether haemoperfusion can alter cytokine, endotoxin or pathogen levels sufficiently to have a biological impact is unclear. A trial that evaluated 97 patients with severe sepsis or septic shock and acute lung injury or ARDS showed that haemoperfusion removed IL-6 from the blood but did not lower circulating IL-6 levels 6 . Overall, the theoretical foundation for the use of haemoperfusion in severe COVID-19 remains limited.

The efficacy of haemoperfusion in patients with COVID-19 has yet to be established with trials or even large observational studies ${ }^{4}$. FDA approval for the haemoperfusion devices was based on "bench performance testing and reported clinical experience" ${ }^{\prime \prime}$. More generally, haemoperfusion has been found to be ineffective in reducing mortality in patients with sepsis. An initial small trial suggested improved survival with haemoperfusion for septic shock, but subsequent larger randomized controlled trials (RCTs) suggested no significant mortality benefit ${ }^{7}$. These findings are consistent with the failure of previous efforts to mitigate inflammation using convective modes of KRT in patients with sepsis. Although convective modes of clearance such as haemofiltration and haemodiafiltration are more effective at removing larger molecules, including toxic cytokines, than haemodialysis, this increased effectiveness has not been shown to translate into clinical benefits $^{8}$. We therefore remain sceptical that haemoperfusion will prove to be effective in treating severe COVID-19. Our scepticism has been bolstered by the recent results of the RECOVERY trial ${ }^{2}$ and of the STAART-AKI trial of early versus late initiation of KRT in patients with $\mathrm{AKI}^{9}$.

The patients with COVID-19 who might theoretically benefit from haemoperfusion are the same severely ill group as those who were shown to benefit from dexamethasone in the RECOVERY trial' ${ }^{2}$. However, dexamethasone is a far less expensive, more broadly available and less-invasive immunomodulatory therapy than haemoperfusion, and is now considered to be the standard-of-care for COVID- 19 . To demonstrate that haemoperfusion is effective in severe COVID-19, sufficiently powered trials must now show that this therapy can provide benefits via immunomodulation beyond those that are already provided by dexamethasone.

Although the theoretical benefits of haemoperfusion remain ill-defined, the associated risks are better known (FIG. 1). The results of the STARRT-AKI trial indicate that pre-emptive intervention with KRT (with which haemoperfusion is often employed in tandem) in the absence of emergent indications for this therapy is associated with excess adverse events 9 . Current use of haemoperfusion for COVID-19 may be due to physicians' perceived need to take action in the face of this daunting illness even if the intervention could be futile or potentially harmful ${ }^{10}$. There is a compelling argument that use of uncontrolled interventions during the COVID-19 pandemic, including hydroxychloroquine, convalescent plasma transfusions and haemoperfusion, represents a missed learning opportunity and such interventions "need to be evaluated within the appropriate ethical and legal framework to ensure the benefit of future patients" ${ }^{\prime 1}$.

Despite widespread interest, potential theoretical benefits and approval from regulatory bodies, the use of haemoperfusion in patients with severe COVID-19 is an expensive experimental therapy that seems unlikely to provide much added benefit for patients who are treated with dexamethasone. We are hopeful that haemoperfusion ultimately proves to be an effective rescue therapy for critically ill patients with COVID-19 who do not respond to dexamethasone, but at present we conclude that it should be available for use only in the context of properly designed RCTs that are powered for clinically important outcomes.

\footnotetext{
1. Li, H. et al. SARS-CoV-2 and viral sepsis: observations and hypotheses. Lancet 395, 1517-1520 (2020).

2. Horby, P. et al. Dexamethasone in hospitalized patients with Covid-19 - preliminary report. N. Engl. J. Med. https://doi.org/ 10.1056/NEJMoa2021436 (2020).
} 
3. Ronco, C. \& Reis, T. Kidney involvement in COVID-19 and rationale for extracorporeal therapies. Nat. Rev. Nephrol. 16, 308-310 (2020).

4. Ronco, C. et al. Extracorporeal blood purification and organ support in the critically ill patient during COVID-19 pandemic: expert review and recommendation. Blood Purif. https:// doi.org/10.1159/000508125 (2020).

5. FDA Emergency Use Authorization Approval Documents. Available at: https://www.fda.gov/emergency-preparednessand-response/mcm-legal-regulatory-and-policy-framework/ emergency-use-authorization\#covidothermeddev (2020).

6. Schadler, D. et al. The effect of a novel extracorporeal cytokine hemoadsorption device on IL-6 elimination in septic patients: a randomized controlled trial. PLOS ONE 12, e0187015 (2017).

7. Dellinger, R. P. et al. Effect of targeted polymyxin B

hemoperfusion on 28-day mortality in patients with septic shock and elevated endotoxin level: the EUPHRATES randomized clinical trial. JAMA 320, 1455-1463 (2018).

8. Friedrich, J. O., Wald, R., Bagshaw, S. M., Burns, K. E. \& Adhikari, N. K. Hemofiltration compared to hemodialysis for acute kidney injury: systematic review and meta-analysis. Crit. Care 16, R146 (2012)

9. Bagshaw, S. M. et al. Timing of initiation of renal-replacement therapy in acute kidney injury. N. Engl. J. Med. 383, 240-251 (2020).

10. Arulkumaran, N., Wright, T., Harris, S. \& Singer, M. Uncontrolled interventions during pandemics: a missed learning opportunity? Intensive Care Med. https://doi.org/10.1007/s00134-020-06180-6 (2020).

\section{Competing interests}

The authors declare no competing interests. 